J. Lake Sci. (湖泊科学), 2015, 27(2): 243-249

http: //www. jlakes. org. E-mail : jlakes@niglas.ac.cn

(c) 2015 by Journal of Lake Sciences

\title{
利用水生植物氮同位素作为人为氮输入太湖的生物指标”
}

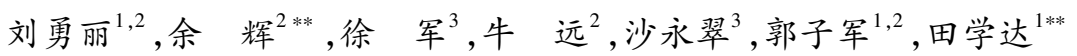

( 1 : 湘潭大学化工学院, 湘潭 411105$)$

( 2 :中国环境科学研究院湖泊生态环境创新基地, 北京 100012)

(3: 中国科学院水生生物研究所,武汉 430072)

摘 要: 富营养化是一个全球性的环境问题, 需要更准确地评估人为氮输人对水生生态系统的影响. 而初级生产者的 $\mathrm{N}$ 同位素已成为一个有用的生物指标. 本研究通过测定太湖和太湖周围湖荡水生植物的 $\delta^{15} \mathrm{~N}$, 与水体中的环境因子做 GAM 分析, 结果表明 $\delta^{15} \mathrm{~N}$ 与水体中的铵态氮、正磷酸盐、总氮都有很显著的相关性, 而这主要是由于植物在吸收和同化过程中 有较大的同位素效应. 水生植物的氮同位素值与氮的有效性和植物对氮的需求有关. 当氮浓度有限时, 植物对氮的需求 变大并且减少了 ${ }^{15} \mathrm{~N}$ 的分馏, 水生植物则有较高的同位素值, 而高氮浓度下, 氮的可用性超过植物对氮的需求, ${ }^{15} \mathrm{~N}$ 分馏增 大则氮同位素值较低. 无锡地区和吴江地区湖荡水生植物同位素值有显著的差异性, 无锡地区湖荡湿地富营养化最严 重, 所以水生植物的 $\delta^{15} \mathrm{~N}$ 值比吴江地区高. 总体来说, 水生植物氮同位素可以作为评价生态系统人为氮输人影响的一个 有效且简单的生物指标.

关键词: 水生植物 $\delta^{15} \mathrm{~N} ; \mathrm{GAM}$; 植物氮的需求; 人为氮输人;生物指标

\section{Stable nitrogen isotope in aquatic macrophytes as an indicator of anthropogenic nitrogen inputs to Lake Taihu}

\author{
LIU Yongli ${ }^{1,2}$, YU Hui $^{2}$, XU Jun ${ }^{3}$, NIU Yuan ${ }^{2}$, SHA Yongcui ${ }^{3}$, GUO Zijun ${ }^{1,2} \&$ TIAN Xueda ${ }^{1}$ \\ (1: College of Chemical Engineering, Xiangtan University, Xiangtan 411105, P. R. China) \\ (2: Research Center of Lake Environment, Chinese Research Academy of Environmental Science, Beijing 100012, P. R. China) \\ (3: Institute of Hydrobiology, Chinese Academy of Sciences, Wuhan 430072, P. R. China)
}

Abstract: Facing the global problem of eutrophication, accurate assessment of the effect of anthropogenic nitrogen inputs to the aquatic ecosystem is needed. The nitrogen isotope in primary producers has become a useful biological index. In our study, we measured the $\delta^{15} \mathrm{~N}$ of aquatic plants in Lake Taihu and surrounding regions, and examined its relationship with water nutrient concentrations with the GAM technique. It is concluded that the $\delta^{15} \mathrm{~N}$ and ammonium, phosphate, total nitrogen in the water column have significant correlations. This is because aquatic plants have larger isotope fractionation during nitrogen absorption and assimilation. The $\delta{ }^{15} \mathrm{~N}$ of aquatic plants is related to plant nitrogen demand and nitrogen availability. When the concentration of nitrogen is limited, the plant demands increase and plants have higher $\delta^{15} \mathrm{~N}$ values consistent with constrained discrimination against the heavier ${ }^{15} \mathrm{~N}$ isotope. When the concentration of nitrogen is higher, where nitrogen availability exceeds plant demand and plants discrimination against the heavier ${ }^{15} \mathrm{~N}$ isotope, the plants have lower $\delta^{15} \mathrm{~N}$ values. There is an extremely significant difference in the $\delta^{15} \mathrm{~N}$ of aquatic plants between Wuxi region and Wujiang region. The water pollution of lakes in Wuxi region was the highest so that the latter have lower $\delta^{15} \mathrm{~N}$ values than the former. The nitrogen isotopes of aquatic plant can be used as an effective and simple biological indicator to evaluate the effect of anthropogenic nitrogen inputs into ecological systems.

Keywords: $\delta^{15} \mathrm{~N}$ of aquatic plants; GAM; plant nitrogen demand; anthropogenic nitrogen inputs; biological indicator

富营养化目前是很多国家面临的一个持久的环境难题, 主要是人口增长、人类活动加快和污染排放增 加等带来的影响. 由于这些区域氮、磷的增加, 湖泊生态系统面临越来越大的压力 ${ }^{[1-2]}$. 随着富营养化的发

* 国家水体污染控制与治理科技重大专项(2012ZX07101-001)资助. 2014-05-19 收稿;2014-07-03 收修改稿. 刘 勇丽 (1989 ), 女, 硕士研究生;E-mail: liuyl1026@ 126. com.

** 通信作者;E-mail: yuhui@ craes. org. cn; snowy@xtu. edu.cn. 
展, 湖泊生态系统将会出现生物多样性下降、生物群落结构单一化、水体质量下降等问题 ${ }^{[3-5]}$. 人为氮输人对 水生生态系统造成不利影响, 需要找到合适的指标来评价水质的变化 ${ }^{[6]}$. 而稳定同位素技术在环境领域中 被认为是很好的天然示踪剂, 在污染物迁移和转化过程中组成稳定, 不造成二次污染, 因此被广泛应用 ${ }^{[7]}$.

初级生产者的 $\delta^{15} \mathrm{~N}$ 来指示氮的来源和氮污染负荷已经不是一个新的技术. 海草、红树林、藻类等植物的 $\delta^{15} \mathrm{~N}$ 都已经被用来评价废水输人对生态系统的影响 ${ }^{[6,8-9]}$. 氮有两种稳定同位素 ${ }^{14} \mathrm{~N}$ 和 ${ }^{15} \mathrm{~N}$, 相对丰度 ${ }^{14} \mathrm{~N}=$ $99.64 \%,{ }^{15} \mathrm{~N}=0.36 \%$. 水生生态系统中氮的来源包括降水、化肥、动物粪便、污水排放、地下水和微生物的氮 循环等,这些来源有不同的 $\delta^{15} \mathrm{~N}$. 例如化肥的 $\delta^{15} \mathrm{~N}$ 为 $-3 \% 0 \sim 3 \%$, 污水或者动物粪便的 $\delta^{15} \mathrm{~N}$ 为 $10 \% 0 \sim 22 \%$, 大气中的 $\delta^{15} \mathrm{~N}$ 为 $2 \% 0 \sim 8 \% 0^{[10-11]}$. 随着时间的推移, 初级生产者将依据他们的增长整合利用这些营养物质, 反映出一定的同位素特征 ${ }^{[8]}$. 水生植物氮同位素已经被证实是一种很好的指示剂, 可以用来指示人为氮的 输人 ${ }^{[12]}$. 水生植物会吸收水中的溶解性无机氮 ( DIN) (包括硝态氮 $\left(\mathrm{NO}_{3}^{-}-\mathrm{N}\right)$ 和铵态氮 $\left(\mathrm{NH}_{4}^{+}-\mathrm{N}\right)$ ), 在吸收和 同化过程具有较大的同位素效应, 被吸收、同化后的氮素比吸收同化前更富集 ${ }^{15} \mathrm{~N}$. 硝化、反硝化、挥发过程均 会产生比较大的同位素分馏 ${ }^{[10]}$. 一些研究表明初级生产者的 $\delta^{15} \mathrm{~N}$ 随污水中 DIN 的增加而增加 ${ }^{[13]}$, 污染区 域发现的植物 $\delta^{15} \mathrm{~N}$ 比未污染区域植物 $\delta^{15} \mathrm{~N}$ 高 ${ }^{[4,14]}$.

太湖流域有 172 条河流连通着太湖 ${ }^{[15]}$, 大多数污染物流入的河流位于西部或西北部, 而污染物流出的 河流位于南部或东南部, 大约有 $30 \% \sim 40 \%$ 的氮、磷被留在湖中 ${ }^{[3]}$. 氮、磷和其他一些元素是植物生长所必需 的, 如果水体收到更多不必要的氮和磷, 生态系统稳定性将改变, 例如发生蓝藻水华. 而外源物质的输人是 造成太湖富营养化的主要原因 ${ }^{[16]}$. 本研究利用同位素技术, 探讨水生植物 $\delta^{15} \mathrm{~N}$ 与水体环境因子之间的关 系, 进而评定水生植物 $\delta^{15} \mathrm{~N}$ 可以作为湖泊输人负荷的指示器, 为太湖外源负荷的监测提供一种有效且简单 的方法, 同时为太湖流域生态评价提供依据.

\section{1 材料与方法}

\section{1 研究区概况}

太湖是我国第三大淡水湖, 位于长江的下游, 跨江苏无锡、苏州和浙江湖州等城市 $\left(30^{\circ} 55^{\prime} 40^{\prime \prime} \sim\right.$ $31^{\circ} 32^{\prime} 58^{\prime \prime} \mathrm{N}, 119^{\circ} 52^{\prime} 32^{\prime \prime} \sim 120^{\circ} 36^{\prime} 10^{\prime \prime} \mathrm{E}$ ). 湖泊面积 $2338 \mathrm{~km}^{2}$, 湖长 (从北到南) $68.5 \mathrm{~km}$, 湖宽 (从东到西) $56 \mathrm{~km}$. 平均水深 $1.9 \mathrm{~m}$, 最大深度 $2.6 \mathrm{~m}^{[3]}$.

重污染的湖荡主要集中在太湖流域西北部, 即无锡、宜兴、苏州辖区, 污染较轻的湖荡主要集中在太湖 流域东南部, 即湖州辖区 ${ }^{[17]}$. 近 10 年来, 由于城市化加快、水体高密度养殖和污染物排放增加, 太湖夏季蓝 藻水华频繁暴发,湖荡水体生态严重退化,水生生物多样性全面衰退.

\section{2 样品采集与处理}

在太湖以及太湖周边湖荡布置采样点 (图 1), 用采水器在水表层 $0 \sim 0.5 \mathrm{~m}$ 采集水样. 采集的水样在冷 藏条件下运回实验室, 通过 GF/C 玻璃纤维滤膜 $(47 \mathrm{~mm}$ ) 过滤后的滤液转移到 $250 \mathrm{ml}$ 瓶子中, 滤膜用于叶绿 素和悬浮物的测定, 滤液用于测定其他水化指标. 载有悬浮物的滤膜经过 $105^{\circ} \mathrm{C}$ 烘干后, 用电子天平称量悬 浮物 (SS) 的质量, 该值与水样体积的比值即为悬浮物的浓度. 叶绿素 a (Chl. a) 采用丙酮萃取分光光度法测 定; 高锰酸盐指数 $\left(\mathrm{COD}_{\mathrm{Mn}}\right)$ 采用高锰酸盐指数法测定; 总氮 $(\mathrm{TN})$ 采用碱性过硫酸钾消解紫外分光光度法测 定; $\mathrm{NH}_{4}^{+}-\mathrm{N}$ 采用纳氏试剂比色法测定; 总磷 ( TP) 采用过硫酸钾消解钼酸铵分光光度法测定; 正磷酸盐 $\left(\mathrm{PO}_{4}^{3-}\right)$ 采用钼酸铵分光光度法测定 ${ }^{[18]}$.

水生植物样品用采草器在水样采样点的周围采集, 采集的植物种类包括挺水植物、沉水植物、浮叶植物、 自由漂浮植物. 采集的每株植物取其最新叶片放人塑料袋, 带回实验室处理. 将其表面附着藻刮洗后, 用去离 子水反复冲洗 3 次, 在 $60^{\circ} \mathrm{C}$ 下烘干 $48 \mathrm{~h}$ 至恒重, 用研钭研磨成均匀粉末后放人细菌保存管中保存.

\section{3 稳定同位素分析}

植物样品分析所用的仪器为中国科学院水生生物研究所 Carlo Erba EA-1110 元素分析仪与 Delta Plus Finnigan 同位素比率质谱连用仪. 氮同位素比值以 $\delta$ 值的形式表达: $\delta^{15} \mathrm{~N}=\left[\left(R_{\text {sample }} / R_{\text {standard }}\right)-1\right] \times 1000$. 式 中, $R_{\text {sample }}$ 为所测得的同位素比值, 氮同位素是 ${ }^{14} \mathrm{~N} /{ }^{15} \mathrm{~N} ; R_{\text {standard }}$ 为标准物质的同位素比值, 氮稳定同位素测定 的标准物质为 $\mathrm{N}_{2} . \delta$ 值越小表示样品重同位素 ${ }^{15} \mathrm{~N}$ 含量越低, 越大表示样品重同位素 ${ }^{15} \mathrm{~N}$ 含量越高. 每测定 10 


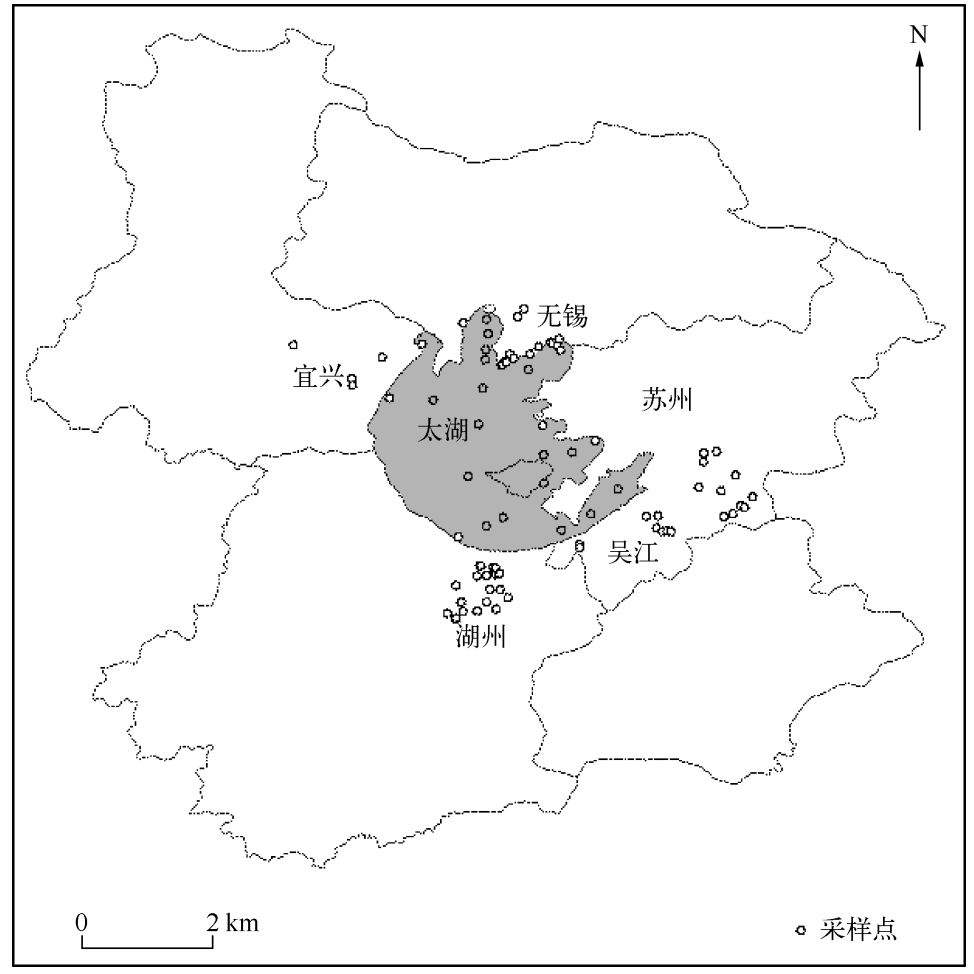

图 1 太湖地区采样点分布

Fig. 1 Location of sampling sites in Lake Taihu regions

个样品插人 1 个标准样品,并随机挑选 $1 \sim 2$ 个样品复测. 样品分析精度为 $\pm 0.3 \%{ }^{[19]}$.

\section{4 数据处理}

如果数据不符合正态分布, 选择数据转换也未能实现正态分布, 则使用 Kruskal-Wallis 检验 (秩和检验) 差异显著性,样本间的多重比较则使用 Kruskalmc 检验. 如果数据符合正态分布,则使用 $t$ 检验来检验差异显 著性.

由于水生植物 $\delta^{15} \mathrm{~N}$ 与水体环境因子之间的关系复杂, 不能用多元线性回归解释它们之间的相互关系, 所以用广义可加模型 GAM 来探讨. GAM 为广义线性模型的非参数化扩展,其优点是能直接处理响应变量与 多个解释变量之间的非线性关系. 广义可加模型在拟合响应变量与解释变量之间的非线性关系过程中, 需 考虑曲线的拟合优度和光滑度, 还要避免或者减轻过度拟合. 根据 GAM 中得出的各解释变量的显著程度 $(P$ 值) 和赤池信息量基准 ( AIC 值) 来对模型变量进行选择,篮选出最优的解释变量.

本研究中, $\delta^{15} \mathrm{~N}$ 为响应变量, 并且进行对数转换, 以太湖和太湖湖荡水体各个因子 $\mathrm{SS} 、 \mathrm{COD}_{\mathrm{Mn}} 、 \mathrm{NH}_{4}^{+}-\mathrm{N}$ 、 $\mathrm{TN} 、 \mathrm{TP} 、 \mathrm{PO}_{4}^{3-} 、 \mathrm{Chl} . \mathrm{a}$ 为解释变量, 采样时间 (time) 作为随机变量加人到模型中, 建立 GAM 模型:

$$
\log \left(\delta^{15} \mathrm{~N}\right)=s(\mathrm{SS})+s(\mathrm{TN})+s\left(\mathrm{NH}_{4}^{+}-\mathrm{N}\right)+s(\mathrm{TP})+s\left(\mathrm{PO}_{4}^{3-}\right)+s\left(\mathrm{COD}_{\mathrm{Mn}}\right)+s(\text { Chl. a })+s(\text { time })+\varepsilon
$$

式中, $s$ 为自然样条平滑, $\varepsilon$ 为误差项. AIC 值越小, 表明模型的拟合效果越佳, 利用 $P$ 值判定各因子的显著性 是否存在差异.

\section{2 结果}

\section{1 稳定同位素分析结果}

太湖水生植物的 $\delta^{15} \mathrm{~N}$ 组成在 $3.5 \% 0 \sim 25 \%$ 之间变化,其中水鳖的 $\delta^{15} \mathrm{~N}$ 值最大 $(16.59 \% 0 \pm 4.18 \% 0)$, 金银 莲花的 $\delta^{15} \mathrm{~N}$ 值最小 $(7.29 \% 0 \pm 0.95 \%$ ). 采集到的植物中, 分布较多的植物种类为沉水植物, 有竹叶眼子菜、 
菹草、穗花狐尾藻、金鱼藻等, 自由漂浮植物较少, 仅有少量的水鳖和槐叶苹. 挺水植物主要有芦苇、菰、空心 莲子草 (表 1). 不同生活型的 $\delta^{15} \mathrm{~N}$ 值没有显著性差异 $(P>0.05)$, 黑藻的 $\delta^{15} \mathrm{~N}$ 值和其他水生植物之间没有 显著性差异 $(P>0.05)$.

\section{表 1 太湖采集植物 $\delta^{15} \mathrm{~N}$ 值的概括统计 *}

Tab. 1 Summary statistics of $\delta^{15} \mathrm{~N}$ values for the macrophytes collected in Lake Taihu

\begin{tabular}{|c|c|c|c|c|c|c|c|}
\hline \multirow{2}{*}{ 科 } & \multirow{2}{*}{ 种名 } & \multirow{2}{*}{ 生活型 } & \multicolumn{5}{|c|}{$\delta^{15} \mathrm{~N}$} \\
\hline & & & $n$ & 平均值 $/ \% 0$ & 最小值 $/ \% 0$ & 最大值 $/ \%$ o & 标准差 $/ \% 0$ \\
\hline 苋科 & 空心莲子草 Alternanthera philoxeroides & 2 & 13 & 13.71 & 6.46 & 21.11 & 4.82 \\
\hline 金鱼藻科 & 金鱼藻 Ceratophyllum demersum & 1 & 18 & 13.15 & 4.62 & 22.67 & 5.59 \\
\hline 小二仙草科 & 穗花狐尾藻 Myriophyllum spicatum & 1 & 29 & 12.42 & 7.49 & 19.05 & 2.43 \\
\hline \multirow[t]{3}{*}{ 水鳖科 } & 黑藻 Hydrilla verticillata & 1 & 6 & 11.86 & 7.02 & 25.63 & 6.87 \\
\hline & 水鳖 Hydrocharis dubia & 4 & 10 & 16.59 & 11.45 & 25.52 & 4. 18 \\
\hline & 苦草 Vallisneria natans & 1 & 8 & 12.32 & 9.04 & 18.15 & 3.29 \\
\hline \multirow[t]{2}{*}{ 睡菜科 } & 金银莲花 Nymphoides indica & 3 & 4 & 7.29 & 6.25 & 8.50 & 0.95 \\
\hline & 荇菜 Nymphoides peltatum & 3 & 31 & 12.10 & 4.39 & 21.91 & 4.11 \\
\hline \multirow[t]{3}{*}{ 禾本科 } & 芦苇 Phragmites australis & 2 & 47 & 11.11 & 3.51 & 20.13 & 3.63 \\
\hline & 荩草 Arthraxon hispidus & 2 & 2 & 13.40 & 10.77 & 16.03 & 3.72 \\
\hline & 菰 Zizania latifolia & 2 & 34 & 12.72 & 4.86 & 23.37 & 4. 40 \\
\hline \multirow[t]{3}{*}{ 眼子菜科 } & 菹草 Potamogeton crispus & 1 & 39 & 13.89 & 8.06 & 20.34 & 3.32 \\
\hline & 篦齿眼子菜 Potamogeton pectinatus & 1 & 5 & 13.80 & 12.47 & 15.23 & 1.02 \\
\hline & 竹叶眼子菜 Potamogeton wrightii & 1 & 64 & 12.68 & 6.75 & 21.94 & 3.11 \\
\hline 菱科 & 菱 Trapa natans & 3 & 15 & 11.40 & 4.20 & 15.25 & 3.16 \\
\hline 香蒲科 & 香蒲 Typha orientalis & 2 & 3 & 10.91 & 8.93 & 13.49 & 2.34 \\
\hline 槐叶苹科 & 槐叶苹 Salvinia natans & 4 & 2 & 10.35 & 10.25 & 10.45 & 0.14 \\
\hline
\end{tabular}

$*$ 表中所示有生活型 $2=$ 挺水植物, $1=$ 沉水植物, $3=$ 浮叶植物, $4=$ 自由漂浮植物, $n$ 为样品数量.

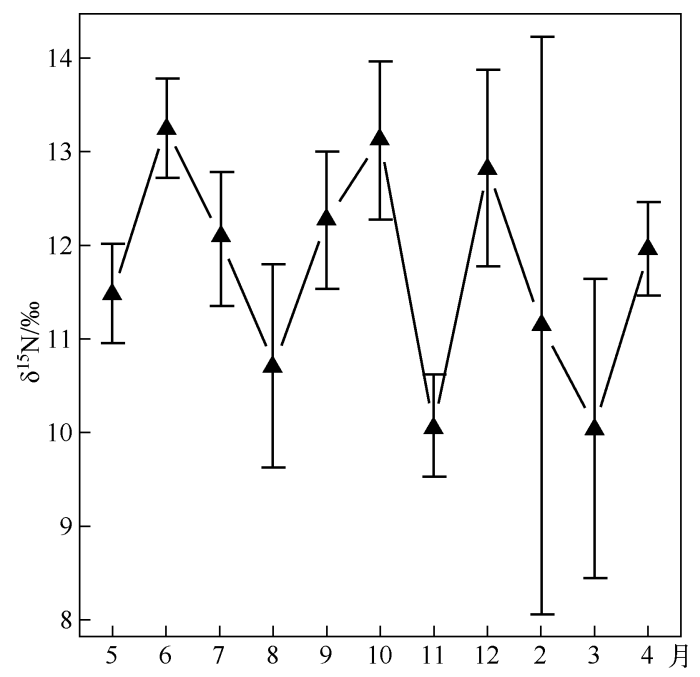

图 2 太湖水生植物样品稳定氮同位素比值随 取样时间的月变化趋势

Fig. 2 Monthly trend of stable nitrogen isotope ratios in aquatic plant samples from Lake Taihu through the sampling time
由图 2 可知, 太湖各个月份水生植物 $\delta^{15} \mathrm{~N}$ 值之间有显著差异性 (Kruskal 检验, $P<$ $0.05) .6$ 月份水生植物的 $\delta^{15} \mathrm{~N}$ 平均值最高, 而 11 月份 $\delta^{15} \mathrm{~N}$ 最小. 11 月和 6 月水生植物的 $\delta^{15} \mathrm{~N}$ 有显著差异 $(P<0.05)$, 其余月份之间的 $\delta^{15} \mathrm{~N}$ 没有显著差异 $(P>0.05)$. 按照北半球平均气 温将太湖地区的四季做如下划分: 11 月下旬至 次年 2 月底为冬季; 3 月上旬至 5 月中旬为春 季; 5 月底至 9 月上旬为夏季; 9 月中旬至 11 月 中旬为秋季 ${ }^{[20]}$. 结果表明季节之间的 $\delta^{15} \mathrm{~N}$ 没 有显著变化 (Kruskal 检验, $P=0.334$ ).

\section{2 水生植物稳定同位素与环境因子的 GAM 模型分析}

GAM 模型结果表明, 有 5 个因子进人模 型, 模型的总解释率为 $25.8 \%, R^{2}$ 为 0.236 . 其 中 $\delta^{15} \mathrm{~N}$ 与 $\mathrm{PO}_{4}^{3-}$ 相关性极显著 $(P$ 值远远小于 $0.001), \mathrm{TN} 、 \mathrm{Chl} . \mathrm{a} 、 \mathrm{TP} 、 \mathrm{NH}_{4}^{+}-\mathrm{N}$ 与 $\delta^{15} \mathrm{~N}$ 显著相 关 $(P<0.05)$. 由图 3 所示, $\mathrm{PO}_{4}^{3-}$ 与水生植物 $\delta^{15} \mathrm{~N}$ 存在非线性关系, 对 $\delta^{15} \mathrm{~N}$ 影响最大, $\delta^{15} \mathrm{~N}$ 随着 $\mathrm{PO}_{4}^{3-}$ 浓度的增大而增大. $\mathrm{PO}_{4}^{3-}$ 浓度在 $0.015 \sim 0.025 \mathrm{mg} / \mathrm{L}$ 时, $\delta^{15} \mathrm{~N}$ 值下降, 大于 0.025 
$\mathrm{mg} / \mathrm{L}$ 时, $\delta^{15} \mathrm{~N}$ 值单调递增. $\mathrm{TN}$ 浓度小于 $2.5 \mathrm{mg} / \mathrm{L}$ 时, 对于 $\delta^{15} \mathrm{~N}$ 值影响不大, 大于 $2.5 \mathrm{mg} / \mathrm{L}$ 时, $\delta^{15} \mathrm{~N}$ 值单调 递减. $\mathrm{NH}_{4}^{+}-\mathrm{N}$ 浓度和水生植物 $\delta^{15} \mathrm{~N}$ 存在显著的正相 关,且为线性关系. TP、Chl. a 对于 $\delta^{15} \mathrm{~N}$ 值变化影响 较小.

\section{3 讨论}

研究结果表明, 不同生活型的水生植物 $\delta^{15} \mathrm{~N}$ 没有 显著差异, 可能是由于采集的都是水里的新叶, 在吸收 同化过程中都发挥了等同的同位素效应. Madsen 等 ${ }^{[21]}$ 发现沉水植物去掉根部后, 叶子也可以对水中营养物 进行吸收, 并且叶子对水体中营养物的吸收能力强于 沉积物 ${ }^{[22]}$. 已有研究表明, 黑藻具有典型的 $\mathrm{C}_{4}$ 型光合 作用途径 ${ }^{[23]}$, 但是其 $\delta^{15} \mathrm{~N}$ 值与其他 $\mathrm{C}_{3}$ 途径植物比较 没有显著性差异, 也说明了水生植物氮同位素不受其 生活型和光合反应途径的影响.

本研究中水生植物 $\delta^{15} \mathrm{~N}$ 与水体中的 $\mathrm{NH}_{4}^{+}$呈线性 正相关关系. 水生植物会吸收水体中的 $\mathrm{DIN}\left(\mathrm{NH}_{4}^{+}-\mathrm{N}\right.$ 和 $\left.\mathrm{NO}_{3}^{-}-\mathrm{N}\right), \mathrm{NH}_{4}^{+}-\mathrm{N}$ 吸收相比于 $\mathrm{NO}_{3}^{-}-\mathrm{N}$ 吸收能降低能 量消耗, 因此 $\mathrm{NH}_{4}^{+}-\mathrm{N}$ 成为许多初级生产者的最优先氮 源 ${ }^{[24]}$. 水生植物对 $\mathrm{NO}_{3}^{-} 、 \mathrm{NH}_{4}^{+}$等无机盐的吸收和同化 过程具有较大的同位素效应. 氨挥发过程能产生 ${ }^{15} \mathrm{~N}$ 贫化的 $\mathrm{NH}_{3}$, 使剩余的 $\mathrm{NH}_{4}^{+}-\mathrm{N}$ 富集 ${ }^{15} \mathrm{~N}$. 硝化过程同样 使剩余的 $\mathrm{NH}_{4}^{+}-\mathrm{N}$ 富集 ${ }^{15} \mathrm{~N}$, 植物吸收这些富集的 ${ }^{15} \mathrm{~N}$ 导 致高的 $\delta^{15} \mathrm{~N}$. 这一结论只适用水体中 $\mathrm{NH}_{4}^{+}-\mathrm{N}$ 浓度较低 时, 本研究中 $\mathrm{NH}_{4}^{+}-\mathrm{N}$ 浓度为 $0 \sim 2 \mathrm{mg} / \mathrm{L}$, 但当 $\mathrm{NH}_{4}^{+}-\mathrm{N}$ 浓度较高时,植物 $\delta^{15} \mathrm{~N}$ 的变化可能相反. Cole 等 ${ }^{[13]}$ 发 现随着地理位置改变,一些植物 $\delta^{15} \mathrm{~N}$ 伴随着水体中 DIN 浓度增加而增加. 所以本研究中水生植物 $\delta^{15} \mathrm{~N}$ 的 变化规律能很好地指示水体中人为氮输人的变化.

水体中 $\mathrm{TN}$ 的浓度与 $\delta^{15} \mathrm{~N}$ 呈负相关. 可能有以下 几个原因: (1) 氮源发生变化, 不同来源的 $\delta^{15} \mathrm{~N}$ 不同, 污水或者动物粪便的 $\delta^{15} \mathrm{~N}$ 为 $10 \% 0 \sim 22 \%$, 化肥污水的 $\delta{ }^{15} \mathrm{~N}$ 为 $-3 \% 0 \sim 3 \%$, 同化过程中的分馏程度随氮的来 源变化 ${ }^{[25]}$. 吸收氮源中重的 ${ }^{15} \mathrm{~N}$ 导致水生植物 $\delta^{15} \mathrm{~N}$ 值 偏高, 吸收贫化的 ${ }^{15} \mathrm{~N}$ 导致水生植物 $\delta^{15} \mathrm{~N}$ 偏低; (2) 随 着 $\mathrm{TN}$ 浓度的升高, 氮的可用性超过了氮的需求, 不是 所有可利用的氮都能被植物吸收, 氮的吸收则会更倾 向 ${ }^{14} \mathrm{~N}$ 而不是 ${ }^{15} \mathrm{~N},{ }^{15} \mathrm{~N}$ 分馏从而导致水生植物 $\delta^{15} \mathrm{~N}$ 值 减小. 湿生植物 ${ }^{[26]}$ 、浮游植物 ${ }^{[27]}$ 、水生植物和红树 林 ${ }^{[28-29]}$ 植物叶子的 $\delta^{15} \mathrm{~N}$ 与氮的有效性和植物对氮的 需求之间的关系已经被研究, 结果都表明植物的氮同 位素特征与氮的可用性和植物对氮的需求有关.
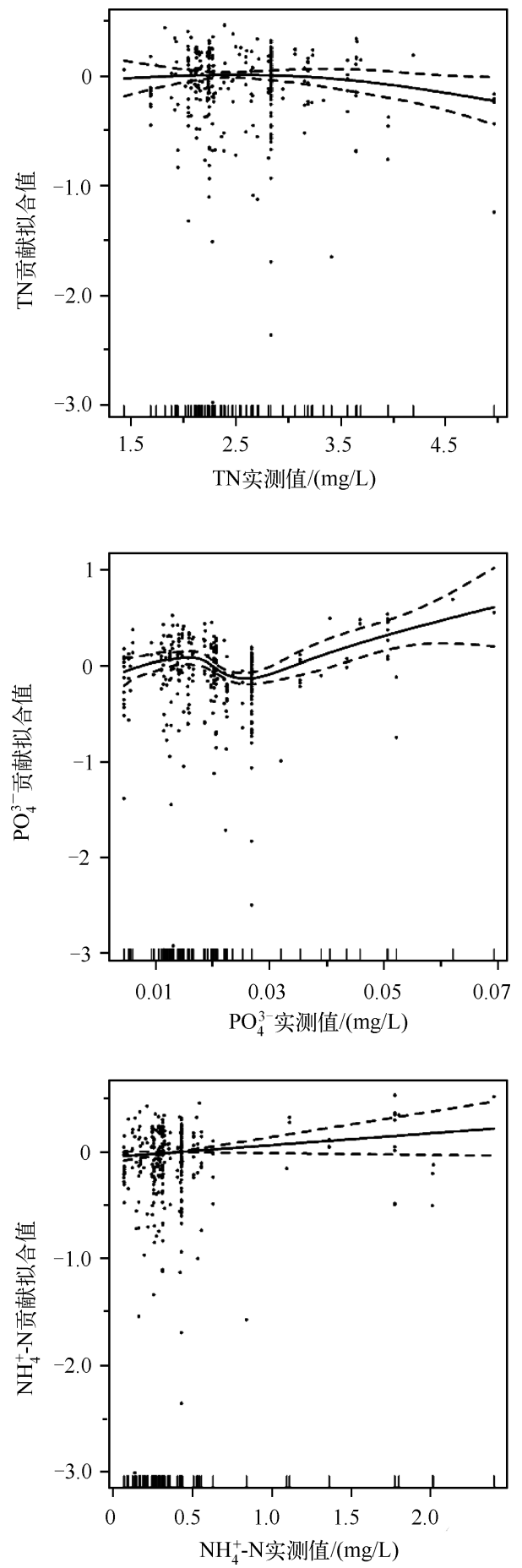

图 3 水生植物 $\delta^{15} \mathrm{~N}$ 与水体环境因子 $\mathrm{GAM}$ 分析 (实线表示氮同位素与各因子关系的加性函 数,虚线表示拟合加性函数逐点的标准差)

Fig. 3 Relationship between aquatic plants nitrogen isotopic signatures and its environmental parameters in water column from GAM 
水生植物的 $\delta^{15} \mathrm{~N}$ 随着 $\mathrm{PO}_{4}^{3-}$ 浓度增加, 总体上呈现上升的趋势. 外部 $\mathrm{P}$ 可用性的变化已经被证实会影响 红树林的 $\delta^{15} \mathrm{~N}^{[26]}$, 这一理论同样适用于水生植物 ${ }^{[30-31]}$. 磷浓度较低时水生植物有较低的 $\delta^{15} \mathrm{~N}$. 沉积物中的 磷释放造成水体中无机磷浓度很高 ${ }^{[32]}$, 此时氮是主要的限制因子. 磷的浓度增加, 导致植物对氮的需求变大 并且减少了 ${ }^{15} \mathrm{~N}$ 的分馏,所有的氮同位素都能够被吸收, 因此植物的 $\delta^{15} \mathrm{~N}$ 很高.

有研究表明, 2000-2007 年相对于无锡地区, 吴江地区城镇建设用地较少 ${ }^{[33]}$, 而城镇建设用地对水质 指标 (溶解氧、 $\mathrm{COD}_{\mathrm{Mn}}$ 和 $\mathrm{NH}_{4}^{+}-\mathrm{N}$ ) 有负面影响 ${ }^{[34]}$. 无锡区域重富营养化的湖荡就占有 $26.7 \%$, 而苏州、湖州、 常进地区只存在中富营养和富营养湖荡. 在无锡地区湖荡采集植物样品数量 $n=52$, 吴江地区为 $n=28$, 对两 个地区的水生植物 $\delta^{15} \mathrm{~N}$ 值进行比较, 结果表明有显著性差异 $(t$ 检验, $P<0.05)$, 无锡地区湖荡水生植物的 $\delta^{15} \mathrm{~N}$ 值较吴江地区湖荡的高. Fry 等 ${ }^{[29]}$ 发现红树林叶子的 $\delta^{15} \mathrm{~N}$ 值在受人为影响较大的红树林与受人为影响 较小的氮输人区之间有显著的差异性, 前者 $\delta^{15} \mathrm{~N}$ 值较高, 后者则较低. 这些研究都说明了水生植物能够很好 地指示外源输人对水生生态系统的影响.

\section{4 结论}

本研究结果表明, 水生植物氮同位素值不受生活型和光合反应途径的影响, 主要与氮的有效性和植物 对氮的需求有关 ${ }^{[35-36]}$. 利用水生植物 $\delta^{15} \mathrm{~N}$ 值作为指示器可以成功地评估人为氮输人对水生生态系统的影 响, 这个研究结果提供了一个简单的工具去评价外源营养物质输人对湖泊生态系统的影响, 而不是用传统 的监测水体营养物质的方法, 为评价太湖流域水环境提供理论依据.

\section{5 参考文献}

[ 1 ] Vitousek PM, Mooney HA, Lubchenco J et al. Human domination of Earth's ecosystems. Science, 1997, 277 (5325) : 494499.

[ 2 ] Smith VH, Tilman GD, Nekola JC. Eutrophication: impacts of excess nutrient inputs on freshwater, marine, and terrestrial ecosystems. Environmental Pollution, 1999, 100(1/2/3) : 179-196.

[ 3 ] Qin B, Xu P, Wu Q et al. Environmental issues of Lake Taihu, China. Hydrobiologia, 2007, 581 (1) : 3-14.

[ 4 ] Benson ER, O' Neil JM, Dennison WC. Using the aquatic macrophyte Vallisneria americana (wild celery) as a nutrient bioindicator. Hydrobiologia, 2008, 596(1) : 187-196.

[ 5 ] Qin B, Gao G, Zhu G et al. Lake eutrophication and its ecosystem response. Chinese Science Bulletin, 2013, 58 (9) : 961 970 .

[ 6 ] Viana IG, Bode A. Stable nitrogen isotopes in coastal macroalgae: Geographic and anthropogenic variability. Science of the Total Environment, 2013, 443: 887-895.

[ 7 ] 白志鹏,张利文,朱 坦等. 稳定同位素在环境科学研究中的应用进展. 同位素, 2007,20(1):57-64.

[ 8 ] Schubert PR, Karez R, Reusch TB et al. Isotopic signatures of eelgrass (Zostera marina L. ) as bioindicator of anthropogenic nutrient input in the western Baltic Sea. Marine Pollution Bulletin, 2013, 72(1) : 64-70.

[ 9 ] Costanzo SD, Udy J, Longstaff B et al. Using nitrogen stable isotope ratios $\left(\delta^{15} \mathrm{~N}\right)$ of macroalgae to determine the effectiveness of sewage upgrades: changes in the extent of sewage plumes over four years in Moreton Bay, Australia. Marine Pollution Bulletin, 2005, 51(1/2/3/4) : 212-217.

[10] Heaton T. Isotopic studies of nitrogen pollution in the hydrosphere and atmosphere: a review. Chemical Geology: Isotope Geoscience Section, 1986, 59: 87-102.

[11] Kreitler CW, Jones DC. Natural soil nitrate: The cause of the nitrate contamination of ground water in Runnels County, Texasa. Ground Water, 1975, 13(1) : 53-62.

[12] Kohzu A, Miyajima T, Tayasu I et al. Use of stable nitrogen isotope signatures of riparian macrophytes as an indicator of anthropogenic N inputs to river ecosystems. Environmental Science and Technology, 2008, 42(21) : 7837-7841.

[13] Cole ML, Kroeger KD, Tomasky GL et al. Assessment of a $\delta^{15} \mathrm{~N}$ isotopic method to indicate anthropogenic eutrophication in aquatic ecosystems. Journal of Environmental Quality, 2004, 33(1) : 124-132.

[14] Grice A, Loneragan N, Dennison W. Light intensity and the interactions between physiology, morphology and stable isotope ratios in five species of seagrass. Journal of Experimental Marine Biology and Ecology, 1996, 195(1) : 91-110. 
[15] 许朋柱,秦伯强. 2001-2002 水文年环太湖河道的水量及污染物通量. 湖泊科学, 2005,17(3):213-218.

[16] 胡开明,李 冰,王 水等. 太湖流域 (江苏省) 水质污染空间特征. 湖泊科学, 2014,26(2):200-206.

[17] 徐泽新. 太湖流域湖荡湿地沉积物碑隶的空间分布及污染评价 [学位论文].武汉:华中农业大学,2013.

[18］国家环境保护总局《水和废水监测分析方法》编委会. 水和废水监测分析方法: 第四版. 北京: 中国环境科学出版 社,2002.

[19] Xu J, Cao T, Zhang M et al. Isotopic turnover of a submersed macrophyte following transplant: the roles of growth and metabolism in eutrophic conditions. Rapid Communications in Mass Spectrometry, 2011, 25(21): 3267-3273.

[20] 饶加旺,马荣华,段洪涛等. 太湖上空大气气溶胶光学厚度及其特征分析. 环境科学, 2012,33(7):2158-2164.

[21] Madsen TV, Cedergreen N. Sources of nutrients to rooted submerged macrophytes growing in a nutrient-rich stream. Freshwater Biology, 2002, 47(2) : 283-291.

[22] Brabandere LD, Frazer TK, Montoya JP. Stable nitrogen isotope ratios of macrophytes and associated periphyton along a nitrate gradient in two subtropical, spring-fed streams. Freshwater Biology, 2007, 52(8) : 1564-1575.

[23] 苏睿丽,李 伟. 沉水植物光合作用的特点与研究进展. 植物学通报,2006,22(S1): 128-138.

[24] Nichols DS, Keeney DR. Nitrogen nutrition of Myriophyllum spicatum: uptake and translocation of ${ }^{15} \mathrm{~N}$ by shoots and roots. Freshwater Biology, 1976, 6(2) : 145-154.

[25] Kendall C, McDonnell JJ. Isotope tracers in catchment hydrology. Eos, Transactions American Geophysical Union, 1999, $\mathbf{8 0}(23): 260$.

[26] Clarkson BR, Schipper LA, Moyersoen B et al. Foliar ${ }^{15} \mathrm{~N}$ natural abundance indicates phosphorus limitation of bog species. Oecologia, 2005, $144(4)$ : 550-557.

[27] Goericke R, Montoya J, Fry B. Physiology of isotopic fractionation in algae and cyanobacteria. In: Stable isotopes in ecology and environmental science. Blackwell, 1994 : 187-221.

[28] McKee KL, Feller IC, Popp M et al. Mangrove isotopic $\left(\delta^{15} \mathrm{~N}\right.$ and $\left.\delta^{13} \mathrm{C}\right)$ fractionation across a nitrogen vs. phosphorus limitation gradient. Ecology, 2002, 83(4) : 1065-1075.

[29] Fry B, Bern AL, Ross MS et al. $\delta^{15} \mathrm{~N}$ and $\delta^{13} \mathrm{C}$ Studies of Nitrogen Use by the Red Mangrove Rhizophora mangle L. in South Florida. Estuarine, Coastal and Shelf Science, 2000, 50(2) : 291-296.

[30] Jones R, King L, Dent M et al. Nitrogen stable isotope ratios in surface sediments, epilithon and macrophytes from upland lakes with differing nutrient status. Freshwater Biology, 2004, 49(4) : 382-391.

[31] King L, Maberly SC, De Ville MM et al. Nitrogen stable isotope ratios of lake macrophytes in relation to growth form and nutrient-limitation. Fundamental and Applied Limnology, 2009, 175 (4) : 307-315.

[32] 金相灿, 王圣瑞, 庞 燕. 太湖沉积物磷形态及 $\mathrm{pH}$ 值对磷释放的影响. 中国环境科学,2005,24(6):707-711.

[33］张殷俊,陈 爽,彭立华. 平原河网地区水质与土地利用格局关系. 资源科学, 2009,31(12):2150-2156.

[34] 李平星,孙 伟. 改革开放以来苏南地区城市扩展格局与驱动机理研究. 长江流域资源与环境, 2013,22 (12): 1529-1536.

[35] Evans RD. Physiological mechanisms influencing plant nitrogen isotope composition. Trends in Plant Science, 2001, 6(3) : $121-126$.

[36] Xu J, Zhang M. Primary consumers as bioindicator of nitrogen pollution in lake planktonic and benthic food webs. Ecological Indicators, 2012, 14(1): 189-196. 